\title{
Barium Titanate Nanoparticles Formed by Chlorine-Free Ambient Condition Sol Process Using Tetrabutylammonium Hydroxide
}

\author{
Wooje Han, ${ }^{1}$ Byungwook Yoo, ${ }^{2}$ and Hyung-Ho Park ${ }^{1}$ \\ ${ }^{1}$ Department of Materials Science and Engineering, Yonsei University, Seoul 03722, Republic of Korea \\ ${ }^{2}$ Flexible Display Research Center, Korea Electronics Technology Institute, Seongnam 13509, Republic of Korea \\ Correspondence should be addressed to Hyung-Ho Park; hhpark@yonsei.ac.kr
}

Received 5 February 2016; Revised 6 April 2016; Accepted 20 April 2016

Academic Editor: Edward A. Payzant

Copyright ( $\odot 2016$ Wooje Han et al. This is an open access article distributed under the Creative Commons Attribution License, which permits unrestricted use, distribution, and reproduction in any medium, provided the original work is properly cited.

Barium titanate $\left(\mathrm{BaTiO}_{3}: \mathrm{BTO}\right)$ nanoparticles (NPs) were synthesized by chlorine-free ambient condition sol (ACS) process using heat reflux at low temperature of $90^{\circ} \mathrm{C}$. The size distribution and morphology of BTO NPs were investigated by varying the concentration of tetrabutylammonium hydroxide (TBAH). The crystalline size of BTO NPs was decreased with increasing the amount of TBAH capping agent (average size changes from 54.3 to $38.7 \mathrm{~nm}$ for 0 to $0.5 \mathrm{M}$ TBAH in X-ray diffraction measurement). The particle size of BTO NPs was principally controlled by a synthetic control of butyl chain of TBAH and also a steric effect of excess amount of TBAH. The dielectric constant of BTO NPs was decreased from 152 to 144 at $1 \mathrm{MHz}$ after an adoption of TBAH capping agent with almost uniform dielectric loss $(<0.027)$. But the dielectric constant of BTO NPs synthesized with various molar ratio of TBAH $(0.1,0.3$, and 0.5$)$ did not show a distinguished decrease. At the particle size range in this experiment, the dielectric behavior of BTO NPs was found to be mainly dependent on the TBAH ligands at BTO NPs formed during capping process, not on the size of BTO NPs.

\section{Introduction}

Barium titanate $\left(\mathrm{BaTiO}_{3}: \mathrm{BTO}\right)$ is the typical perovskite structure material with most extensively studied ferroelectric ceramic due to its high permittivity (high- $k$ ) and ferroelectric properties [1-4]. The applications in the electronics industry include transducer and actuators, high- $k$ dielectric capacitors, and memory applications, which rely on the existence of a spontaneous polarization in the crystal unit cell. BTO is suitable to sensors, thermistors, multilayer ceramic capacitor, electrooptical devices, and so on as the universal electronic ceramics. In the above application studies, a thin dielectric layer has been investigated for the miniaturization and enhanced dielectric performance [5-7].

The perovskite ceramics have been known that their size, shape, crystallinity, and surface composition are strongly dependent on the synthesis process and physical structure [8-10]. The typical synthesis of nanoparticles (NPs) and wet chemical methods such as hydrothermal and sol-gel process have been extensively studied to synthesize NPs of BTO with high purity. Sol-gel method has been receiving a great deal of attention due to the relative ease, reliability, and homogeneity in the synthesis and processing of nanomaterials with high degree of purity. The use of metal alkoxides as a precursor in sol-gel process has more benefits than solid state reaction $[11,12]$ and hydrothermal process because of molecular level control of structure and composition [13, 14]. However, during sol-gel process, noncrystalline BTO was obtained at room temperature and annealing treatment converts it to crystalline BTO [15-17]. But the heat treatment usually leads to an agglomeration of particles, which increases the size of particles. Although the conventional hydrothermal method makes it possible to directly synthesize spherical BTO with smaller size powders $(<1 \mu \mathrm{m})$, at lower reaction temperature than sol-gel method, it requires relatively harsh reaction conditions and therefore needed special and expensive precursors capable of withstanding at high $\mathrm{pH}$ and temperature as well as pressure [11-17]. 
Freshly, ambient condition sol (ACS) process developed by Wang et al. proposed solution to the above problems [18]. It is possible to control the agglomeration of nanoparticles by low temperature heat treatment, but to obtain uniform particle size, sol-gel process is highly beneficial. ACS process is a useful technology which has an advantage of being able to obtain a high purity and homogeneity of the material. Applying metal alkoxides as a precursor, they have more advantages than solid state reaction and hydrothermal processing, due to a facial adoption of structure and composition control at molecular level [18-20]. Normally chlorine-based precursors have been used for the formation of BTO NPs through ACS process $[18,21]$. In the case of alkoxides precursors, small value of dielectric constant was only reported as around 18 [22].

Tetrabutylammonium hydroxide (TBAH) is the chemical compound with the formula $\left(\mathrm{C}_{4} \mathrm{H}_{9}\right)_{4} \mathrm{NOH}$ with the acronym $\mathrm{TBAOH}$ or TBAH. This species is not readily obtainable as a pure compound, but it is employed as a solution in water or alcohol. It is commonly used as a base in organic chemistry. Relative to more conventional inorganic bases, such as $\mathrm{KOH}$ and $\mathrm{NaOH}, \mathrm{TBAH}$ is more soluble in organic solvents. TBAH is a strong base that is used often under phase-transfer conditions to affect alkylation and deprotonations. TBAH can be neutralized with a variety of mineral acids to give lipophilic salts of the conjugate base. The stability of the NPs could be improved by long-chain effect with butyl chain and strong acidity. These NPs can be repeatedly isolated and redispersed in aqueous solution. As the solubility and stability of these TBAH-stabilized particles are closely linked to the double-layer structure and $\mathrm{pH}$ of the system, 10-12 full characterization is essential for their successful utilization in future applications $[23,24]$.

In this study, the morphology and size distribution of BTO NPs depending on the concentration of TBAH were investigated using chlorine-free ACS process. In the synthesis of BTO NPs by ACS process, the barium chloride and titanium chloride precursors have been preferred owing to the ease of handling and inexpensive chemical input $[18,21]$. Nevertheless, the chlorine ions are very difficult to remove and the residual ions may influence the surface and electrical properties of BTO NPs [25]. Alternatively, barium alkoxide and titanium alkoxide have been used as better substitute precursors for obtaining chlorine-free BTO NPs. This could permit us to control the chlorine impurities and suppress the formation of chlorides. We diminished heat treatment temperature and avoided $\mathrm{HCl}$ damage during the formation of BTO NPs through modified ACS without chloride precursor.

\section{Experimental Procedure}

Barium nitrate $\left(\mathrm{Ba}\left(\mathrm{NO}_{3}\right)_{2}, 99.999 \%\right.$ trace metals basis, Sigma-Aldrich), titanium(IV) isopropoxide ( $\mathrm{Ti}\left[\mathrm{OCH}\left(\mathrm{CH}_{3}\right)_{2}\right]_{4}$, TTIP, reagent grade, 97\%, Sigma-Aldrich), deionized (DI) water, and sodium hydroxide $(\mathrm{NaOH}$, reagent grade, $\geq 98 \%$, pellets (anhydrous)) were used as starting materials, solvent, and reacting agents, respectively. Tetrabutylammonium hydroxide $\left(\left(\mathrm{CH}_{3} \mathrm{CH}_{2} \mathrm{CH}_{2} \mathrm{CH}_{2}\right)_{4} \mathrm{~N}(\mathrm{OH}) \cdot 30 \mathrm{H}_{2} \mathrm{O}, \quad \geq 99.0 \%\right.$,

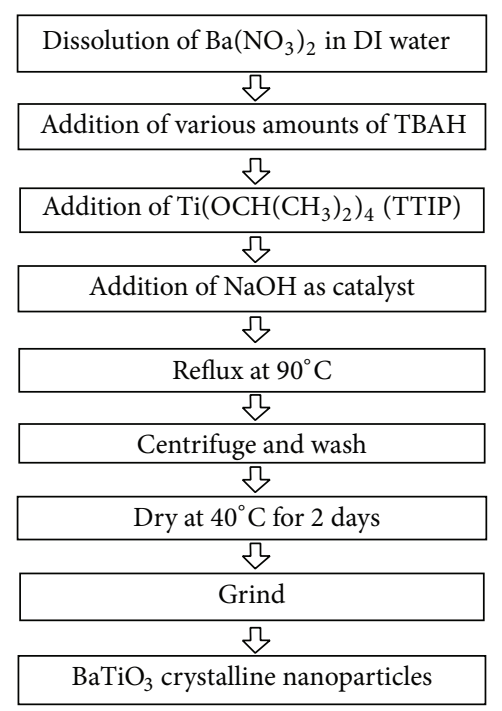

FIGURE 1: Schematic flow chart for synthesis procedure of BTO NPs.

Sigma-Aldrich) was used as capping agent without any purification. The experiments were performed according to recently proposed ACS process [18]. A schematic of experimental process was shown in Figure 1. Initially, barium nitrate as a $\mathrm{Ba}$ precursor was dissolved in water. TBAH was added to $\mathrm{Ba}$ precursor solution and TTIP was added as a Ti precursor to the solution. $\mathrm{NaOH}$ was added as a catalyst to the mixed solution. The molar ratio of $\mathrm{Ba}$ and $\mathrm{Ti}$ precursor was kept constant at $3: 2$, and the concentration of Ti precursor was performed with $0.2 \mathrm{~mol} / \mathrm{L}$. A cloudy solution was formed after mixing of precursors, capping agent, and catalyst. This solution was refluxed at $90^{\circ} \mathrm{C}$ for $20 \mathrm{~min}$ in air atmosphere. The separated particles by using a centrifuge were washed several times using distilled water and butanol to remove the remaining organic materials and impurities. For nanoparticles washing, nanoparticles were precipitated at first by using centrifuge and they were washed with $n$-butanol and water using vortex mixer and sonication. Even insoluble materials can become temporarily dispersed by vortex mixer and sonication which supply strong physical energy for mixture state. Washing is performed by this physical dispersion [26, 27]. Butanol has been also used as a washing solvent to remove impurities in the synthesis of oxide nanoparticles using chlorine-based precursors [28]. In this experiment, $n$-butanol could remove any possibly formed organics matter and impurities, and the aqueous salts and excess capping agents were removed by water washing. The washing of BTO NPs was completed by repeating more than 3 times for each solvent. Finally, particles of BTO NPs were dried at $40^{\circ} \mathrm{C}$ for $48 \mathrm{~h}$ in oven at air atmosphere.

After synthesis of barium titanate nanoparticles, Fourier transform-infrared spectroscopy (FT-IR, PerkinElmer, USA) was employed for the confirmation of capping agent and barium titanate nanoparticles in the precipitate and final product. Crystalline phases and chemical composition were examined by X-ray diffraction (XRD, Ultima model, Rigaku, Japan). For the analysis, $\mathrm{Cu} \mathrm{K} \alpha$ radiation with a wavelength 

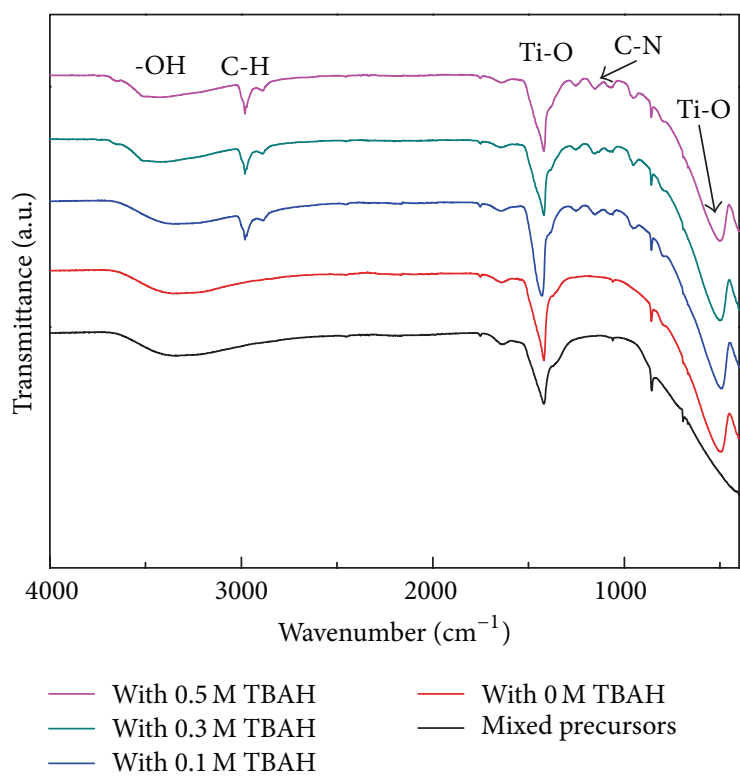

FIGURE 2: FT-IR spectra of BTO NPs obtained before heat refluxing and with various TBAH molar ratios $(0,0.1,0.3$, and 0.5$)$.

of $1.541 \AA$ at $2 \theta$ values ranging from 20 to $80^{\circ}$ was applied. Field emission scanning electron microscopy (FE-SEM, JSM600F, JEOL, Japan) and particles size analyzer (PSA, Nanotrac Wave, Microtrac, USA) were used to observe the morphology and distribution of nanoparticles. The dielectric properties of BTO NPs were measured with pelletized BTO. The porosity of pellet was calculated by measuring the weight and volume of the pellet. The capacitance-voltage and leakage current (4294A, Agilent, USA) behaviors were measured by a dielectric test fixture (16451B, Agilent) to analyze the dielectric properties of BTO NPs.

\section{Results and Discussion}

To study the ligand attachment to TBAH capping agent, FTIR measurements were carried out on the barium titanates synthesized with various TBAH concentrations $(0.1,0.3$, and $0.5 \mathrm{M}$ ). The FT-IR spectra of barium titanates with various TBAH concentrations showed almost the same absorption behavior as given in Figure 2. The absorption peak around $2800-2900 \mathrm{~cm}^{-1}$ was attributed to the $\mathrm{C}-\mathrm{H}$ bond due to the presence of TBAH. The peaks around $1200 \mathrm{~cm}^{-1}$ were attributed to $\mathrm{CH}_{2}$ and $\mathrm{CH}_{3}$ bending modes [20]. Absorption peak of hydroxyl group was observed at $3400 \mathrm{~cm}^{-1}$ in all samples [29]. The Ti-O bonding peak was observed at $480 \mathrm{~cm}^{-1}$ after forming by reflux. At precipitation of mixed precursors, this peak was not observed. The absorption peak at $490 \mathrm{~cm}^{-1}$ is due to the presence of Ti-O in octahedral structure $[30,31]$. This octahedral Ti-O absorption peak is a proof of BTO phase formation with perovskite structure.

When the capping ligands coordinate to the metal ions, the head groups of the capping agent point toward the surface and the tail groups toward the aqueous medium $[13,15$, 20]. TBAH caps the surface of Ba precursor through the

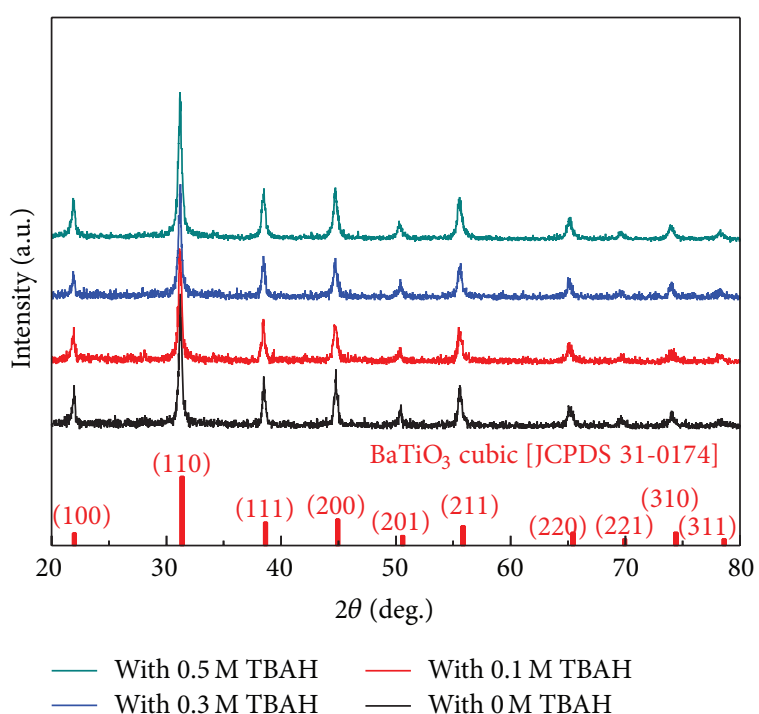

FIGURE 3: XRD spectra of BTO NPs prepared with various TBAH molar ratios $(0,0.1,0.3$, and 0.5$)$.

$\mathrm{N}$ atom [32] and forms a bilayer of capping agent around the nanoparticle's surface [33]. The $\mathrm{N}^{+}$group of TBAH was leading a dipole moment with Ba precursor. The Coulombic interaction between $\mathrm{Ba}$ precursor and $\mathrm{N}^{+}$group of TBAH affects the nucleation and growth of BTO NPs. As a result, the size and distribution of nanoparticles could be controlled by the concentration of TBAH.

The crystalline phase of BTO NPs was analyzed using XRD. The diffraction patterns in Figure 3 were obtained with BTO NPs prepared with various TBAH concentrations. (100), (110), (111), (200), (201), (211), (220), (221), (310), and (311) diffraction peaks were observed. The presence of crystalline BTO diffraction peaks represents the fact that TBAH did not affect the formation of crystalline BTO with cubic structure by refluxing in air atmosphere at low temperature $\left(90^{\circ} \mathrm{C}\right) . \mathrm{NaOH}$ plays as a catalyst during the formation of BTO crystalline phase. Normally high $\mathrm{NaOH}$ (above $10 \mathrm{M} \mathrm{NaOH}$ ) concentration is needed for the formation of crystalline oxides [34-36], but only 1 3 M $\mathrm{NaOH}$ is needed under TBAH condition. The secondary crystalline phase such as $\mathrm{Ba}_{2} \mathrm{TiO}_{4}$ and $\mathrm{BaCO}_{3}$ was not observed and only crystalline phase was $\mathrm{BaTiO}_{3}$ through all conditions.

Scherrer's equation [37] given as below was used to calculate the crystalline size of BTO from the major diffraction peak (110) in the XRD spectra:

$$
D=\frac{K \lambda}{\beta \cos \theta},
$$

where $\beta$ is the width of the observed diffraction line at its halfintensity maximum, $K$ is the shape factor, set to 0.9 , and $\lambda$ is the wavelength of the $\mathrm{Cu} K \alpha$. The crystalline size of BTO NPs was obtained for 0 (pure BTO NPs), 0.1, 0.3, and 0.5 M TBAH concentrations as $54.3,43.4,41.2$, and $38.7 \mathrm{~nm}$, respectively. As TBAH concentration increases, the size of BTO NPs was decreased. 
TABLE 1: Measured and calculated values of dielectric constant of BTO NPs in pelletized form.

\begin{tabular}{|c|c|c|c|c|c|c|c|c|}
\hline \multirow{3}{*}{$\begin{array}{l}\text { Air volume fraction (\%) } \\
\text { Frequency }\end{array}$} & \multicolumn{2}{|c|}{ Pristine BTO } & \multicolumn{2}{|c|}{ With $0.1 \mathrm{M}$ TBAH } & \multicolumn{2}{|c|}{ With $0.3 \mathrm{M}$ TBAH } & \multicolumn{2}{|c|}{ With $0.5 \mathrm{M}$ TBAH } \\
\hline & & & & & & & & \\
\hline & Measured & Calculated & Measured & Calculated & Measured & Calculated & Measured & Calculated \\
\hline $1 \mathrm{kHz}$ & $94 \pm 1$ & $156 \pm 2$ & $74 \pm 1$ & $149 \pm 2$ & $78 \pm 1$ & $149 \pm 2$ & $74 \pm 1$ & $148 \pm 2$ \\
\hline $10 \mathrm{kHz}$ & $93 \pm 1$ & $154 \pm 2$ & $73 \pm 1$ & $148 \pm 2$ & $78 \pm 1$ & $147 \pm 2$ & $74 \pm 1$ & $146 \pm 2$ \\
\hline $100 \mathrm{kHz}$ & $92 \pm 1$ & $152 \pm 2$ & $72 \pm 1$ & $147 \pm 2$ & $77 \pm 1$ & $146 \pm 3$ & $73 \pm 1$ & $145 \pm 2$ \\
\hline $1000 \mathrm{kHz}$ & $92 \pm 1$ & $152 \pm 2$ & $72 \pm 1$ & $146 \pm 2$ & $77 \pm 1$ & $145 \pm 2$ & $73 \pm 1$ & $144 \pm 2$ \\
\hline
\end{tabular}

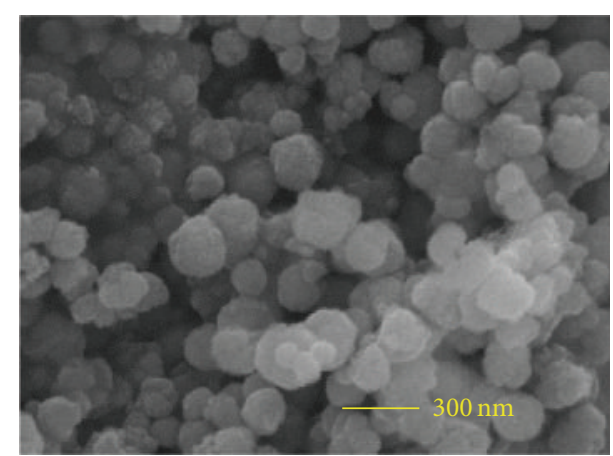

(a)

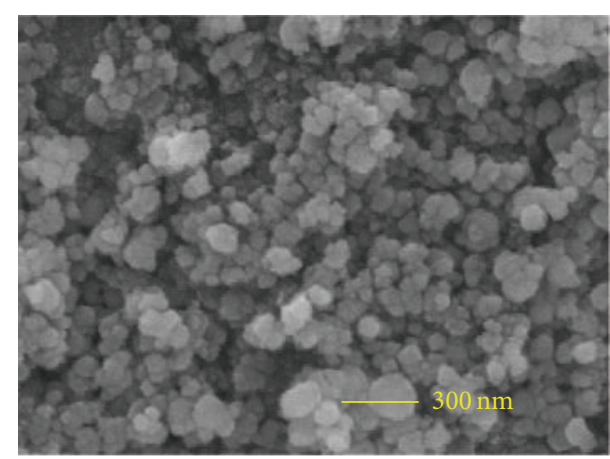

(c)

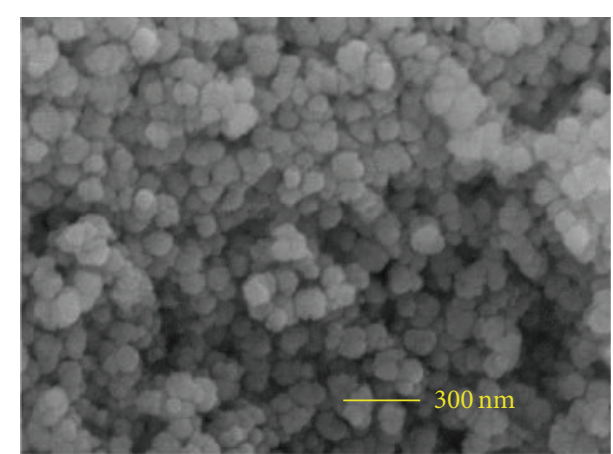

(b)

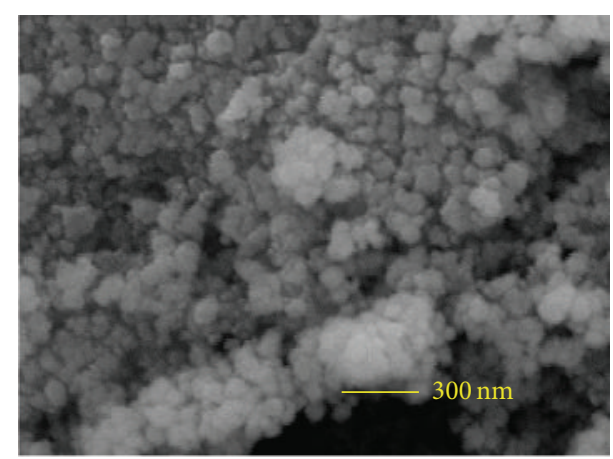

(d)

FIgUre 4: SEM images of BTO NPs synthesized with various TBAH molar ratios: (a) 0, (b) 0.1, (c) 0.3, and (d) 0.5 mol/L TBAH.

The size and morphology of BTO NPs were also characterized by using SEM and PSA and the results were shown in Figures 4 and 5. The size of BTO NPs synthesized at $0-0.5 \mathrm{M}$ TBAH concentration was 300 to $90 \mathrm{~nm}$. The size of BTO nanoparticles measured with SEM was larger than that from the XRD measurement. This might be due to an agglomeration of crystalline BTO NPs and coated bilayer of TBAH because the BTO NP size obtained from XRD measurement corresponds to the crystalline size of the particles. However, the size of BTO NPs showed a decreasing trend with increased TBAH concentration, similar to the results of XRD. Figure 4(a) shows an important agglomeration of primary particles due to an absence of TBAH capping effect. Figures 4(b), 4(c), and 4(d) show the particle size and agglomeration of BTO NPs synthesized at $0.1,0.3$, and $0.5 \mathrm{M}$ TBAH molar ratios, respectively; the size of the NPs was found to be decreased with increased molar ratio of TBAH. PSA results showed the same tendency as decrease in the nanoparticle size with increasing TBAH (Figure 5). The nanoparticles' size was controlled by the coulombic interaction between $\mathrm{Ba}$ precursor and $\mathrm{N}^{+}$group of TBAH during the nucleation and growth of BTO. It is possible to explain that TBAH-stabilized BTO NPs are coated by double layer of capping agent and BTO NPs can exist separately from each other in the solution.

The dielectric constant and dielectric loss of the $\mathrm{BaTiO}_{3}$ nanoparticles synthesized with various TBAH molar ratios as $0,0.1,0.3$, and $0.5 \mathrm{M}$ were measured from $1 \mathrm{kHz}$ to $1,000 \mathrm{kHz}$. For a measurement of dielectric constant of BTO NPs, a complete removal of impurities on nanoparticles except capping agent was confirmed by using FT-IR and they were pelletized. A pelletization is a general method of dielectric measurement of nanoparticles $[38,39]$. The air volume fraction in the pellet is important for a dielectric constant measurement, but as shown in Table 1, the calculated values of air volume fraction did not have any consistent trend. The air volume fraction was calculated for each sample by measuring the volume and weight of each pellet. The dielectric constant of BTO NPs could be calculated by using Lichtenecker's formula as 


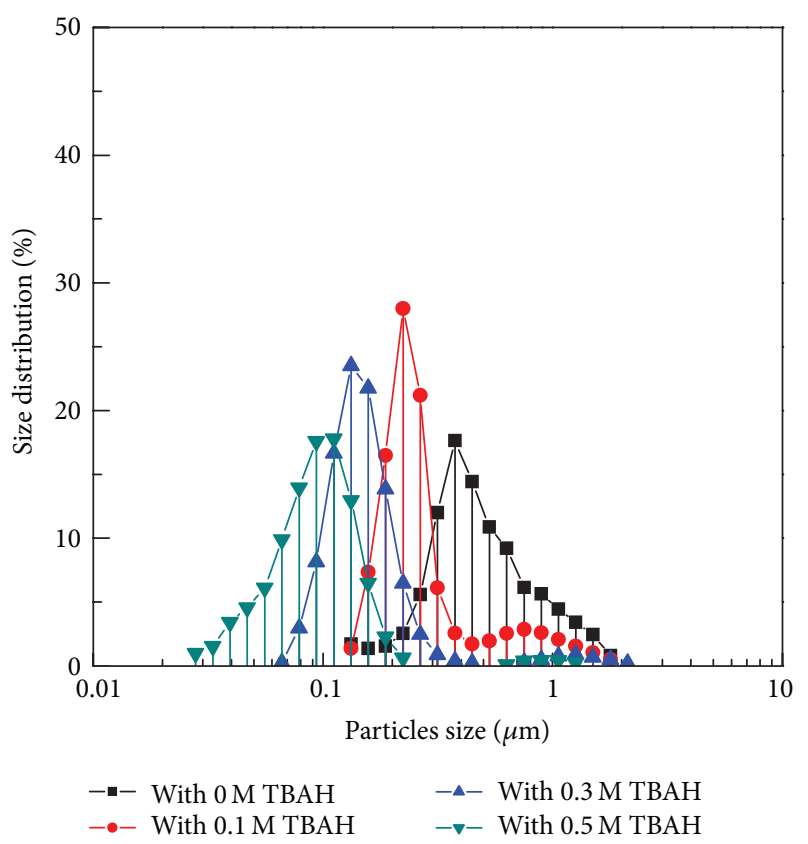

FIgure 5: Particles size distribution of BTO NPs depending on the molar ratio of TBAH.

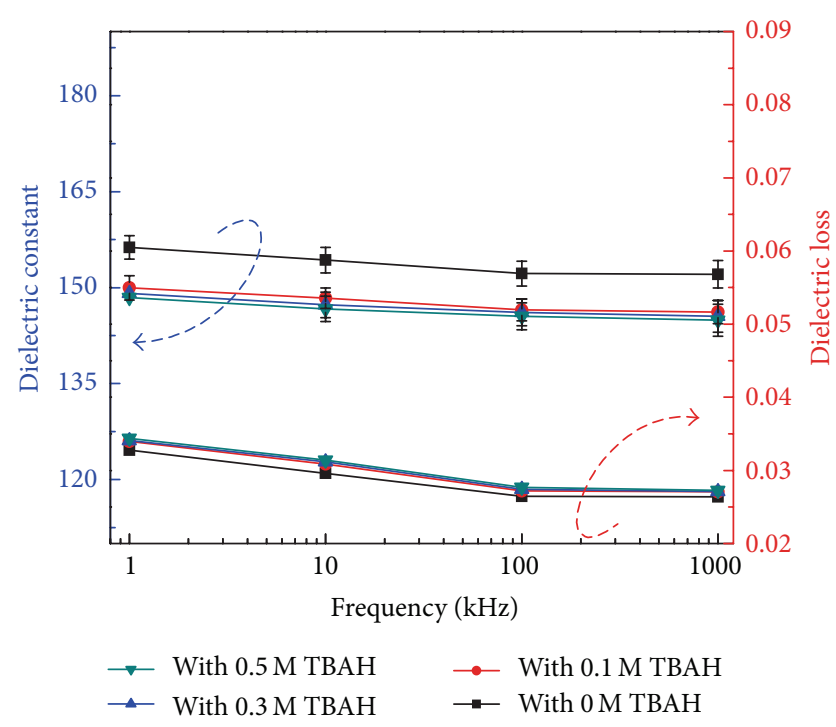

Figure 6: Dielectric properties of BTO NPs synthesized with varying TBAH molar ratios $(0,0.1,0.3$, and 0.5$)$.

$\ln \varepsilon_{\text {total }}=v_{\mathrm{NPs}} \ln \varepsilon_{\mathrm{NPs}}+v_{\text {air }} \ln \varepsilon_{\text {air }}$ [40]. The calculated values were given in Table 1 and Figure 6. The dielectric constant of BTO was decreased by almost 3\% after an addition of TBAH, but not smaller than 144 . Normally the dielectric constant of ferroelectric BTO could be affected by their size. Until some special size of grain, the dielectric constant and size of grain were increased together in nanoscale region. Above peak grain of nanoparticles of the highest dielectric constant, the dielectric constant was reduced [41]. Another reason for a decrease of dielectric constant with decreasing nanoparticle size is an increase of boundary and surface charge compensation layer [42]. However, in this experiment, the dielectric constant of BTO NPs synthesized with various molar ratios of TBAH $(0.1,0.3$, and 0.5$)$ did not show a distinguished decrease with increasing TBAH molar ratio, that is, decreased crystalline size of BTO NPs. A distinguished difference was observed rather between the BTO NPs prepared without and with TBAH capping agent. It means that in this experiment a decrease in dielectric constant of BTO NPs was mainly caused from the presence of TBAH ligands on the BTO NPs, not the crystalline size. However the measured dielectric constant was almost 2 times larger than the reported value for similar size of BTO NPs by Huang et al. [43]. Furthermore BTO NPs showed similar dielectric behaviors regardless of TBAH molar ratio and a similar behavior was also observed in FT-IR results as given in Figure 2, that is, a similar absorption peak position and intensity. From these results, it can be said that NP size control effect of TBAH capping agent is mainly due to a bond formation with BTO NP through capping ligands but also steric effect of TBAH capping agent in the case of increasing TBAH molar ratio. That is to say, due to a steric effect of TBAH, the size of BTO NPs was decreased with increasing TBAH molar ratio; however this extra TBAH showing steric effect should be removed through following centrifuge and washing step. Then, in the FT-IR measurement with dried BTO NPs, an increasing absorption behavior of TBAH-related peak such as $\mathrm{C}-\mathrm{H}$ and $\mathrm{C}-\mathrm{N}$ was not observed with increasing TBAH molar ratio. Also, the dielectric loss was less than 0.035 . From these results, it can be said that crystalline BTO NPs with a high dielectric constant and low dielectric loss could be synthesized using the chlorine-free ACS process with refluxing in an air atmosphere at $90^{\circ} \mathrm{C}$, which is comparable with the values obtained from a thinfilm form of $\mathrm{BaTiO}_{3}$ cubic phase [37].

\section{Conclusion}

The size-controlled BTO NPs were able to be synthesized through chlorine-free ACS process with TBAH as a capping agent under $90^{\circ} \mathrm{C}$ refluxing condition. The size of BTO NPs was decreased by addition of TBAH. TBAH improves the stability of NPs with long-chain effect by butyl chain and strong acidity. These effects induced isolated and dispersed BTO NPs in aqueous solution. In the case of 0.3 and 0.5 molar ratios of TBAH, the amount of TBAH was found to be excess for surface capping of BTO NPs; however the excess amount could induce a reduction in BTO particle size due to a steric effect. The dielectric constant of the crystalline BTO NPs was calculated above 144 with maximum dielectric loss of 0.035 and the dielectric behavior was mainly affected by the presence of TBAH capping ligands and slightly by their size in this experimental condition.

\section{Competing Interests}

The authors declare that they have no competing interests. 


\section{Acknowledgments}

This study was supported by a grant (no. 10041220) from the Fundamental R\&D Program for Core Technology of Materials funded by the Ministry of Knowledge Economy, Republic of Korea.

\section{References}

[1] L. V. Lutsev, A. I. Stognij, and N. N. Novitskii, "Giant magnetoresistance in semiconductor/granular film heterostructures with cobalt nanoparticles," Physical Review B, vol. 80, no. 18, Article ID 184423, 2009.

[2] H. T. Vo and F. G. Shi, "Towards model-based engineering of optoelectronic packaging materials: dielectric constant modeling," Microelectronics Journal, vol. 33, no. 5-6, pp. 409-415, 2002.

[3] E. K. Lee, K. T. Eun, Y. S. Ahn, Y. T. Kim, M. W. Chon, and S. H. Choa, "Alternative sintering technology of printed nanoparticles for roll-to-roll process," Journal of the Microelectronics and Packaging Society, vol. 21, no. 4, pp. 15-24, 2014.

[4] S. C. Gong, H. S. Lim, I. S. Shin et al., "A study of soluble pentacene thin film for organic thin film transistor," Journal of the Microelectronics and Packaging Society, vol. 14, no. 3, pp. 1-6, 2007.

[5] A. Feteira, D. C. Sinclair, I. M. Reaney, Y. Somiya, and M. T. Lanagan, " $\mathrm{BaTiO}_{3}$-based ceramics for tunable microwave applications," Journal of the American Ceramic Society, vol. 87, no. 6, pp. 1082-1087, 2004.

[6] J. A. Davies and A. Dutremez, "Electroceramics from source materials via molecular intermediates: $\mathrm{BaTiO}_{3}$ from $\mathrm{TiO}_{2}$ $\left[\mathrm{Ti}(\text { catecholate })_{3}\right]^{2-}$," Journal of the American Ceramic Society, vol. 73, no. 5, pp. 1429-1430, 1990.

[7] S. Bhattacharjee, M. K. Paria, and H. S. Maiti, "Occurrence of excess titania in strontium titanate prepared by the oxalate precipitation route," Ceramics International, vol. 18, no. 5, pp. 295-300, 1992.

[8] D. K. Hwang, K. Lee, J. H. Kim et al., "Low-voltage highmobility pentacene thin-film transistors with polymer/high- $k$ oxide double gate dielectric," Applied Physics Letters, vol. 88, no. 24, Article ID 243513, 2006.

[9] H. Y. Lee, P. S. Chen, T. Y. Wu et al., "Low power and high speed bipolar switching with a thin reactive Ti buffer layer in robust $\mathrm{HfO}_{2}$ based RRAM," in Proceedings of the IEEE International Electron Devices Meeting, pp. 1-4, San Francisco, Calif, USA, December 2008.

[10] D. E. Kwon, M. H. Azarian, and M. Pecht, "Failure prediction of multilayer ceramic capacitors (MLCCs) under temperaturehumidity-bias testing conditions using non-linear modeling," Journal of the Microelectronics and Packaging Society, vol. 20, no. 3, pp. 7-10, 2013.

[11] T. Yoko, K. Kamiya, and K. Tanaka, "Preparation of multiple oxide $\mathrm{BaTiO}_{3}$ fibres by the sol-gel method," Journal of Materials Science, vol. 25, no. 9, pp. 3922-3929, 1990.

[12] H. B. Sharma and A. Mansingh, "Sol-gel processed barium titanate ceramics and thin films," Journal of Materials Science, vol. 33, no. 17, pp. 4455-4459, 1998.

[13] D. Hennings and S. Schreinemacher, "Characterization of hydrothermal barium titanate," Journal of the European Ceramic Society, vol. 9, no. 1, pp. 41-46, 1992.

[14] E. Ciftci, M. N. Rahaman, and M. Shumsky, "Hydrothermal precipitation and characterization of nanocrystalline $\mathrm{BaTiO}_{3}$ particles," Journal of Materials Science, vol. 36, no. 20, pp. 48754882,2001

[15] I. J. Clark, T. Takeuchi, N. Ohtori, and D. C. Sinclair, "Hydrothermal synthesis and characterisation of $\mathrm{BaTiO}_{3}$ fine powders: precursors, polymorphism and properties," Journal of Materials Chemistry, vol. 9, no. 1, pp. 83-91, 1999.

[16] D. H. Mun and J. S. Ha, "The effect of precursor concentration on $\mathrm{ZnO}$ nanorod grown by low-temperature aqueous solution method," Journal of the Microelectronics and Packaging Society, vol. 20, no. 1, pp. 33-37, 2013.

[17] P. Gherardi and E. Matijević, "Homogeneous precipitation of spherical colloidal barium titanate particles," Colloids and Surfaces, vol. 32, pp. 257-274, 1988.

[18] X. Wang, B. I. Lee, M. Hu, E. A. Payzant, and D. A. Blom, "Nanocrystalline $\mathrm{BaTiO}_{3}$ powder via a sol process ambient conditions," Journal of the European Ceramic Society, vol. 26, no. 12, pp. 2319-2326, 2006.

[19] B. I. Lee, X. Wang, R. Bhave, and M. Hu, "Synthesis of brookite $\mathrm{TiO}_{2}$ nanoparticles by ambient condition sol process," Materials Letters, vol. 60, no. 9-10, pp. 1179-1183, 2006.

[20] C. A. Nolph, D. E. Sievers, S. Kaewgun et al., "Photocatalytic study of polymorphic titania synthesized by ambient condition sol process," Catalysis Letters, vol. 117, no. 3-4, pp. 102-106, 2007.

[21] N. G. Devaraju, B. I. Lee, X. Wang, M. Viviani, and P. Nanni, "Tailoring size of $\mathrm{BaTiO}_{3}$ nanocrystals via ambient conditions sol process," Journal of Materials Science, vol. 41, no. 11, pp. 33353340, 2006.

[22] H. Maie and B. I. Lee, "The hydroxyl concentration and the dielectric properties of barium titanate nano-powder synthesized by water-based ambient condition sol process," Journal of Materials Science: Materials in Electronics, vol. 20, no. 7, pp. 619627, 2009.

[23] A. Mori, T. Kondo, T. Kato, and Y. Nishihara, "Palladiumcatalyzed cross-coupling polycondensation of bisalkynes with dihaloarenes activated by tetrabutylammonium hydroxide or silver(I) oxide," Chemistry Letters, no. 4, pp. 286-287, 2001.

[24] D. M. Djokic, W. B. Stern, and A. Taubert, "Zinc oxide/carbohydrate hybrid materials via mineralization of starch and cellulose in the strongly hydrated ionic liquid tetrabutylammonium hydroxide," Crystal Growth and Design, vol. 8, no. 1, pp. 330-335, 2008.

[25] R. A. De Souza, C. Voisin, H. Schraknepper et al., "Complex diffusion behavior of oxygen in nanocrystalline $\mathrm{BaTiO}_{3}$ ceramics," Physical Chemistry Chemical Physics, vol. 16, no. 6, pp. 25682575, 2014.

[26] G. Dermont, M. Bergeron, G. Mercier, and M. Richer-Laflèche, "Soil washing for metal removal: a review of physical/chemical technologies and field applications," Journal of Hazardous Materials, vol. 152, no. 1, pp. 1-31, 2008.

[27] A. Mizev, A. Trofimenko, D. Schwabe, and A. Viviani, "Instability of marangoni flow in the presence of an insoluble surfactant. Experiments," European Physical Journal: Special Topics, vol. 219, no. 1, pp. 89-98, 2013.

[28] J. B. Mamani, L. F. Gamarra, and G. E. de Souza Brito, "Synthesis and characterization of $\mathrm{Fe}_{3} \mathrm{O}_{4}$ nanoparticles with perspectives in biomedical applications," Materials Research, vol. 17, no. 3, pp. 542-549, 2014.

[29] Y.-S. Her, E. Matijević, and M. C. Chon, "Controlled doublejet precipitation of uniform colloidal crystalline particles of $\mathrm{Zr}$ and Sr-doped barium titanates," Journal of Materials Research, vol. 11, no. 12, pp. 3121-3127, 1996. 
[30] H. Xu, L. Gao, and J. Guo, "Preparation and characterizations of tetragonal barium titanate powders by hydrothermal method," Journal of the European Ceramic Society, vol. 22, no. 7, pp. 1163$1170,2002$.

[31] S. W. Lu, B. I. Lee, and L. A. Mann, "Carbonation of barium titanate powders studied by FT-IR technique," Materials Letters, vol. 43, no. 3, pp. 102-105, 2000.

[32] M. Chakraborty, F.-W. Hsiao, B. Naskar, C.-H. Chang, and A. K. Panda, "Surfactant-assisted synthesis and characterization of stable silver bromide nanoparticles in aqueous media," Langmuir, vol. 28, no. 18, pp. 7282-7290, 2012.

[33] Z. M. Sui, X. Chen, L. Y. Wang et al., "Capping effect of CTAB on positively charged Ag nanoparticles," Physica E: LowDimensional Systems and Nanostructures, vol. 33, no. 2, pp. 308314, 2006.

[34] R. Zanella, S. Giorgio, C.-H. Shin, C. R. Henry, and C. Louis, "Characterization and reactivity in $\mathrm{CO}$ oxidation of gold nanoparticles supported on $\mathrm{TiO}_{2}$ prepared by depositionprecipitation with $\mathrm{NaOH}$ and urea," Journal of Catalysis, vol. 222, no. 2, pp. 357-367, 2004.

[35] Y. Wu, Y. Wang, G. Luo, and Y. Dai, "In situ preparation of magnetic $\mathrm{Fe}_{3} \mathrm{O}_{4}$-chitosan nanoparticles for lipase immobilization by cross-linking and oxidation in aqueous solution," Bioresource Technology, vol. 100, no. 14, pp. 3459-3464, 2009.

[36] J. Zhu, D. Li, H. Chen, X. Yang, L. Lu, and X. Wang, "Highly dispersed $\mathrm{CuO}$ nanoparticles prepared by a novel quick-precipitation method," Materials Letters, vol. 58, no. 26, pp. 33243327, 2004

[37] T. Hoshina, H. Kakemoto, T. Tsurumi, S. Wada, and M. Yashima, "Size and temperature induced phase transition behaviors of barium titanate nanoparticles," Journal of Applied Physics, vol. 99, no. 5, Article ID 054311, 2006.

[38] A. S. Lanje, S. J. Sharma, R. S. Ningthoujam, J.-S. Ahn, and R. B. Pode, "Low temperature dielectric studies of zinc oxide $(\mathrm{ZnO})$ nanoparticles prepared by precipitation method," Advanced Powder Technology, vol. 24, no. 1, pp. 331-335, 2013.

[39] S. Suresh, "Studies on the dielectric properties of CdS nanoparticles," Applied Nanoscience, vol. 4, no. 3, pp. 325-329, 2014.

[40] T. Zakri, J.-P. Laurent, and M. Vauclin, “Theoretical evidence for 'Lichtenecker's mixture formulae' based on the effective medium theory," Journal of Physics D: Applied Physics, vol. 31, no. 13, pp. 1589-1594, 1998.

[41] K. Kinoshita and A. Yamaji, "Grain-size effects on dielectric properties in barium titanate ceramics," Journal of Applied Physics, vol. 47, no. 1, pp. 371-373, 1976.

[42] C. Fang, L. Chen, and D. Zhou, "Influence of domain on grain size effects of the dielectric properties of $\mathrm{BaTiO}_{3}$ nanoceramics and nanoparticles," Physica B: Condensed Matter, vol. 409, no. 1, pp. 83-86, 2013.

[43] L. Huang, Z. Chen, J. D. Wilson et al., "Barium titanate nanocrystals and nanocrystal thin films: synthesis, ferroelectricity, and dielectric properties," Journal of Applied Physics, vol. 100, no. 3, Article ID 034316, 2006. 

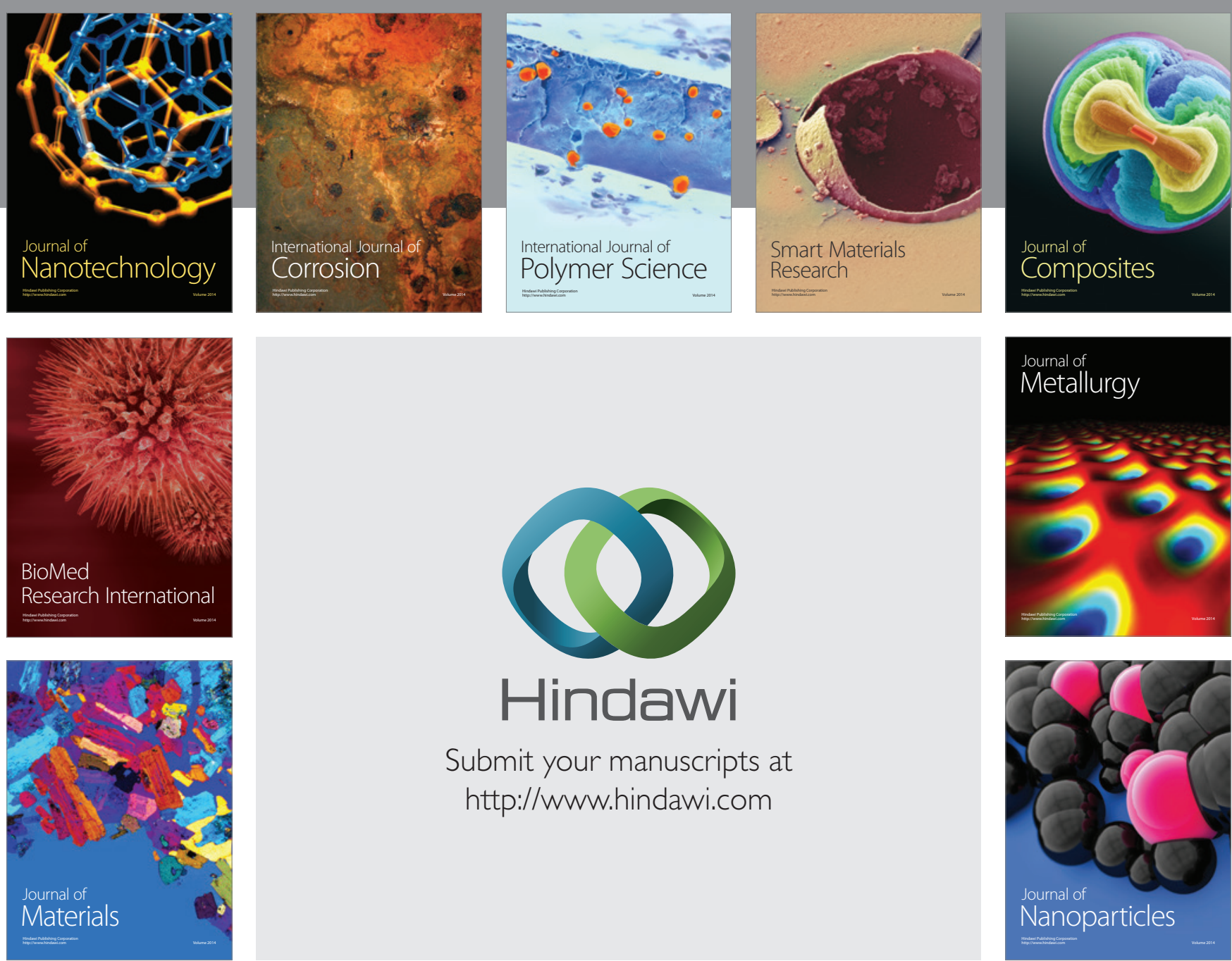

\section{Hindawi}

Submit your manuscripts at

http://www.hindawi.com

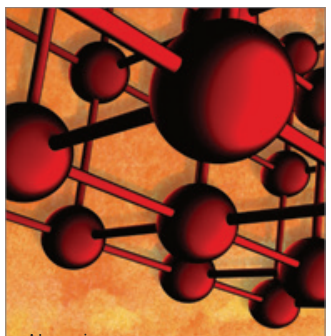

Materials Science and Engineering
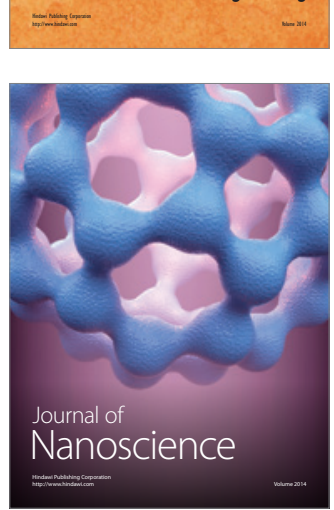
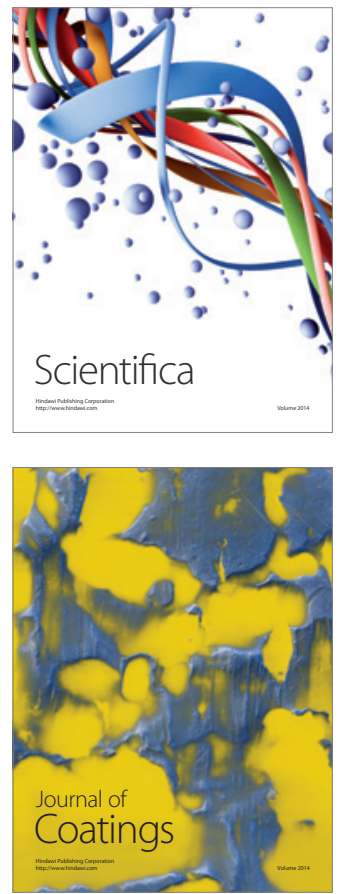
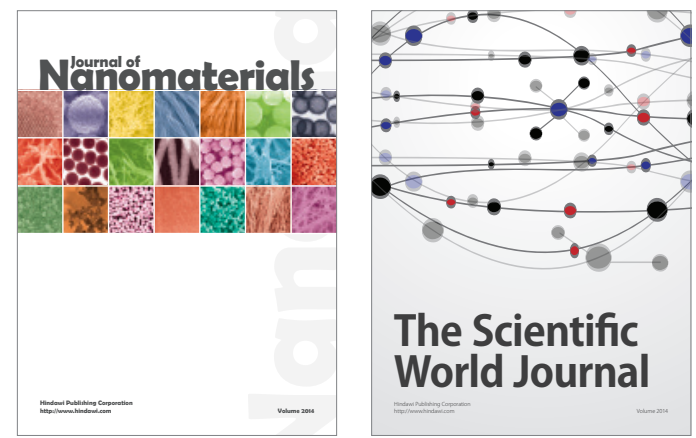

The Scientific World Journal
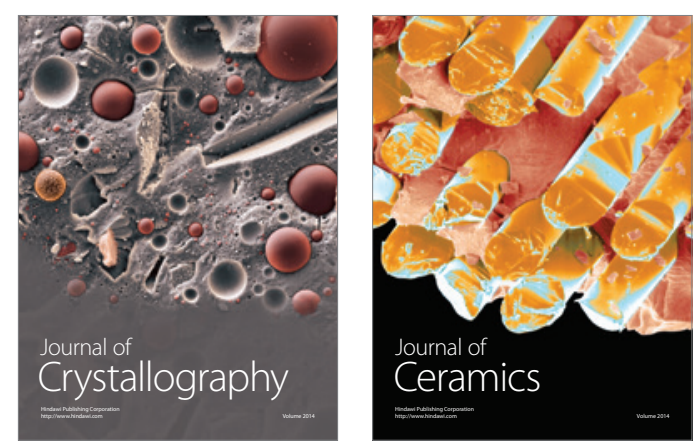
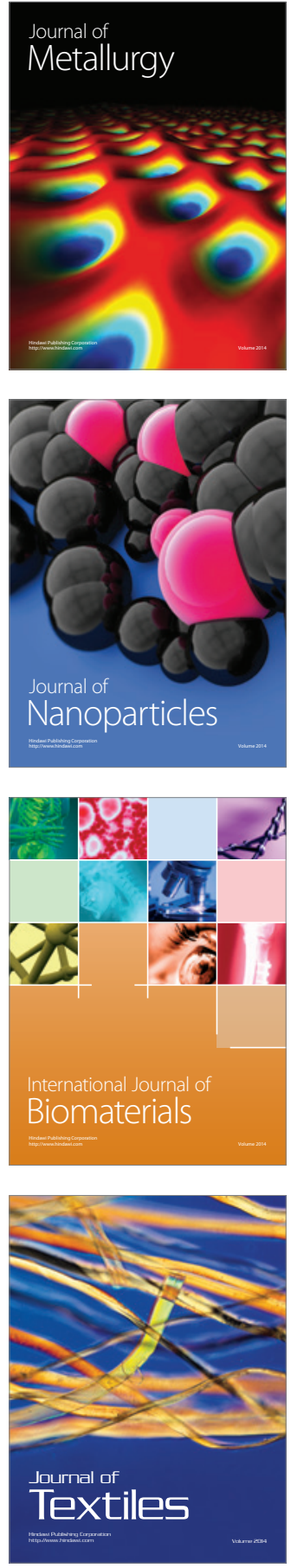